\title{
Végétation et sols climax sur les Grauwackes de la série du Markstein (Hautes-Vosges) (1)
}

\author{
Jean-Pierre BOUDOT, Sylvain BRUCKERT et Bernard SOUCHIER \\ Centre de Pédologie biologique du C.N.R.S. \\ 54500 Vandacuvre-lìs-Nancy
}

\begin{abstract}
Résumé
Sur les Grauwackes du Markstein, l'influence du climat sur la végétation et l'orientation virtuelle de la pédogénèse est la même que dans les Hautes-Vosges granitiques. Cependant on peut observer sur ces matériaux des divergences pédogénétiques assez importantes avec l'évolution sur granite, aux mêmes étages climatiques. L'étagement des sols climax climatiques est le suivant :

"sol brun ocreux » à l'étage de la sapinière-hêtraie (sol brun acide sur granite),

"sol brun acide» à l'étage de la hêtraie culminale (sol brun ocreux et sol ocre podzolique sur granite),

"ranker cryptopodzolique » à l'étage des hautes chaumes.

Sur Grauwackes, l'évolution pédologique est moins marquée que sur granite où elle atteint le stade ocre podzolique. En outre, la répartition altitudinale des sols est inversée aux étages forestiers où, sur granite, on observe la séquence altitudinale " sol brun acide - sol brun ocreux - ranker cryptopodzolique $\gg$. Seul ce dernier type de sol est commun aux deux types de matériaux.
\end{abstract}

\section{I. - Introduction}

On se propose de définir les caractéristiques des stations observées sur les Grauwackes de la Série du Markstein, roches sédimentaires dont le rôle à l'égard de la distribution des groupements floristiques et de la pédogénèse est encore mal connu.

La région considérée est située aux confins des Hautes Vosges centrales et méridionales, dans les hautes vallées de la Lauch et de la Fecht. On y observe un étagement de la végétation et des sols parallèle à celui que l'on rencontre dans les Vosges cristallines (SOuchiER, 1971). Queiques traits importants différencient néanmoins les deux domaines lithologiques et se traduisent par l'apparition sur Grauwacke de climax stationnels originaux et d'un style d'altération pédogénétique propre.

(1) Cette étude a fait l'objet d'une rédaction plus complète qui peut être communiquée sur demande à l'auteur principal. 


\section{II. - Facteurs généraux de l'évolution des stations et des sols}

Cette partie des Vosges possède un climat océanique froid en raison de l'altitude, caractérisé en outre par une faible amplitude thermique et une forte pluviosité. Ces aspects s'accentuent très sensiblement à l'approche des crêtes (température moyenne annuelle : 4 " $\mathrm{C}$; amplitude thermique : $15^{\circ} \mathrm{C}$; pluviométrie moyenne annuelle : 1800 à $2000 \mathrm{~mm}$ ). Celles-ci, généralement orientées Nord-Sud, subissent en effet laction de brouillards fréquents et de vents violents («Effet de crête» décrit par Carbiener, 1963 et 1966).

Ce climat limite l'extension de la végétation forestière à l'approche des sommets et en même temps influe sur la pédogénèse. Les sols deviennent souvent très humifères et sont soumis à une podzolisation climatique au moins potentielle.

En climat montagnard, un deuxième facteur pédogénétique essentiel est la composition de la roche mère et plus exactement celle des matériaux d'altération du substrat géologique (Souchier, 1971). Les grauwackes de la série du Markstein sont des roches sédimentaires primaires de type Flysch, riches en ferromagnésiens notamment en chlorites (GAGNY, 1962) ferrifères. Elles sont ainsi marquées par une importante teneur en fer (3,5 à 9 p. 100, Rivalentı \& Sighinolfi, 1969) facilement altérable. Leur grande gélivité et leur texture fine conduisent à des formations d'altération très pierreuses à matrice riche en limons.

Les formations superficielles comprennent :

1. Des coulées de solifluxion et des dépôts morainiques à texture sablo-limoneuse ou limono-sableuse, recouvrant la plupart des versants. La teneur en argile y oscille entre 4 et 9 p. 100.

2. Des formations d'extension plus réduite dont les caractéristiques sont très particulières : il s’agit des ravins enrichis en argile (20 p. 100), des éboulis dépourvus de terre fine et de cuvettes engorgées.

Au niveau des formations de versants, végétation et sols s'ordonnent suivant un étagement altitudinal régi par le climat et on parle de climax climatiques pour désigner les équilibres sol-végétation (tableau 1).

Au niveau des formations très différenciées, on aura à faire à des climax stationnels (Duchaufour, 1970). A l'étagement altitudinal se superpose en effet une variabilité horizontale, due aux contrastes stationnels liés aux changements des caractéristiques géomorphologiques et topoclimatiques. Dans certains cas ces variations stationnelles (topoclimat) ne font que modifier les limites altitudinales des climax climatiques ou n'induisent que des variations floristiques secondaires. Dans d'autres cas elles ont plus d'influence et on observe des climax stationnels à associations spécialisées. Ce sont alors des facteurs locaux, essentiellement édaphiques, qui déterminent des conditions écologiques très différenciées (roche mère et drainage en particulier...). 


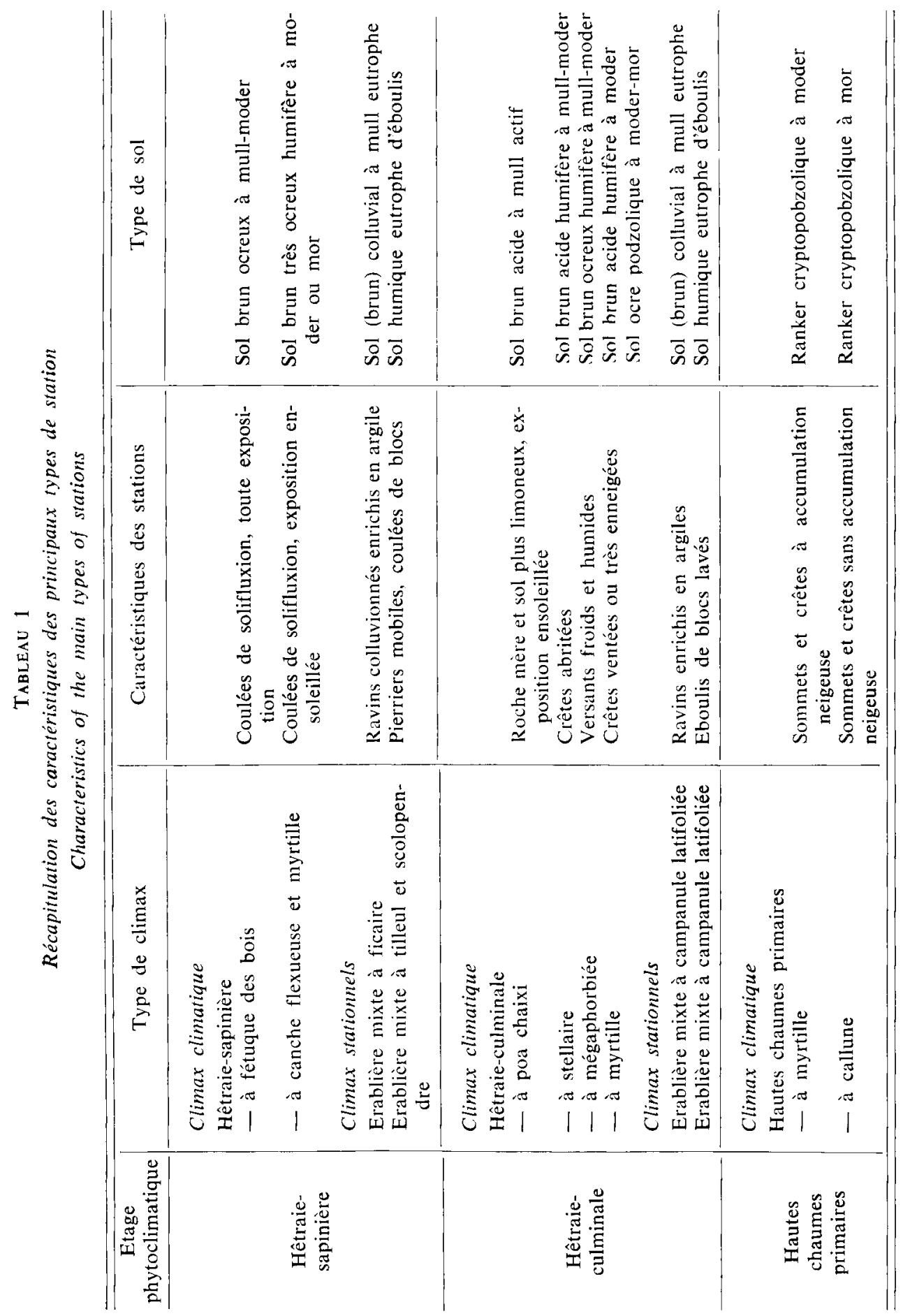


Tableau 2

Composition floristique des associations de la hêtraie-sapinière Floristic composition of plant-associations of the Beech-Fir grove

\begin{tabular}{|c|c|c|}
\hline \multirow{2}{*}{ Espèces } & \multicolumn{2}{|c|}{ Indices d'abondance-dominance extrêmes } \\
\hline & Abieti-fagetum & Luzu!o-fagetum \\
\hline \multicolumn{3}{|l|}{ Strate arborescente } \\
\hline Fagus silvatica $\ldots . \ldots$. & 0 à 5 & 3 à 5 \\
\hline Abies alba $\ldots \ldots \ldots \ldots$ & 0 à 5 & 1 à 5 \\
\hline Acer pseudo platanus... & 0 à + & \\
\hline \multicolumn{3}{|l|}{ Strate herbacée } \\
\hline Festuca silvatica $\ldots \ldots \ldots$. & 0 à 5 & $0 \grave{a}+$ \\
\hline Viola silvestris $\ldots \ldots \ldots \ldots$ & 0 à 2 & \\
\hline Asperula odorata $\ldots \ldots \ldots$ & 0 à 4 & \\
\hline Lamium galeobdolon... . & 0 à 2 & \\
\hline Stellaria nemorum ... & 0 à 1 & \\
\hline Adenostyles albifrons & 0 à 2 & $0 \grave{a}+$ \\
\hline Athyrium filix femina ... & 0 à 1 & $0 \grave{a}+$ \\
\hline Dryopteris filix mas ..... & 0 à 1 & 0 à + \\
\hline Dryopteris dilata $\ldots \ldots \ldots$. & 0 à 1 & \\
\hline Milium effusum $\ldots \ldots \ldots$ & 0 à 1 & \\
\hline Polygonatum verticilat. .... & 0 à 1 & 0 à + \\
\hline Lysimachia nemorum ..... & 0 à 2 & \\
\hline Rubus idaeus $\ldots \ldots \ldots \ldots$. & 0 à 2 & 0 à + \\
\hline Phyteuma spicatum ....... & 0 à 2 & 0 à + \\
\hline Lactuca muralis $\ldots \ldots \ldots$. & 0 à 1 & \\
\hline Deschampsia flexuosa .... & $0 \grave{a} 2$ & 4 à 5 \\
\hline Vaccinium myrtillus . ...... & & 0 à 5 \\
\hline Luzula maxima $\ldots \ldots \ldots$. & $0 \grave{a}+$ & 0 à 5 \\
\hline Melampyrum pratense... & & 0 à 2 \\
\hline Prenanthes purpurea..... & 0 à 2 & $1 \grave{\mathrm{a}} 2$ \\
\hline Senecio nemorensis $\ldots \ldots$. & 0 à 2 & 0 à 1 \\
\hline Luzula albida $\ldots \ldots \ldots \ldots$ & 0 à 2 & 1 à 2 \\
\hline Oxalis acetosella $\ldots \ldots \ldots$ & 1 à 3 & 0 à 1 \\
\hline Hieracium silvaticum $\ldots$. & 0 à 2 & 0 à 2 \\
\hline Galium rotundifolium .... & 0 à + & 0 à 2 \\
\hline Galium asperum $\ldots \ldots$. & 0 à + & 0 à 1 \\
\hline Veronica officinalis $\ldots \ldots$. & 0 à 1 & 0 à + \\
\hline Digitalis purpurea $\ldots \ldots \ldots$ & 0 à + & 0 à 1 \\
\hline Lonicera nigra $\ldots \ldots \ldots \ldots$ & & 0 à + \\
\hline Solidago virga aurea $\ldots .$. & & \\
\hline Euphorbia amygdaloïdes ... & 0 à 2 & \\
\hline Impatiens noli tangere ..... & 0 à 2 & \\
\hline Veronica montana...$\ldots$ & 0 à 2 & \\
\hline
\end{tabular}




\section{III. - Principales stations et type de sols sur Grauwackes}

\section{A. - Les climax climatiques}

La plupart des climax climatiques observés sur Grauwackes ont leur équivalent sur granite, mais nous verrons que leur degré d'évolution reste généralement plus limité. Dans la présentation faite ci-dessous, nous insisterons sur les groupements floristiques et les particularités écologiques qui caractérisent le principaux types de sols. Le détail des descriptions et analyses des stations et des sols est donné en annexe.

Les sols bruns ocreux dont les profils comprennent un horizon (B) de couleur ocre-rouille plus ou moins vive et à structure «foisonnante» en microagrégats inférieurs à $100 \mu$ (Fluffy), représentent les sols climax de l'étage montagnard moyen sous la hêtraie-sapinière à fétuque des bois ou à canche flexueuse et myrtille. Toutefois, le caractère ocreux est moins exprimé sous la fétuque, espèce moins acidiphile que la canche et la myrtille. Les données floristiques de ces deux associations sont rassemblées dans le tableau 2 .

Les facteurs de répartition de la canche et de la fétuque apparaissent complexes. L'association à canche ne se rencontre jamais en exposition Nord; par contre l'association à fétuque est plus indifférente au topoclimat : elle serait plus sensible à l'effet roche mère. On constate en effet que les substrats les plus altérés sont occupés par la fétuque, alors que les moins altérés le sont par la canche et la myrtille (tableau 3) (1), ce qui rejoint les observations faites sur granite (SouCHIER, 1971). Le fait nouveau est que la relation précédente entre degré d'altération et groupes écologiques s'inverse totalement dans les horizons de surface. Sous l'influence du moder, l'altération argilisante devient beaucoup plus forte sous la canche que sous le mull de la fétuque (figure 1) et paradoxalement la canche se retrouve

\section{TABleau 3}

Taux d'altération des horizons $C$ des différents types de station (le fer constitue l'élément de référence)

Weathering rate in $C$ horizons of difjerent types of stations (iron is the reference element)

\begin{tabular}{|c|c|c|c|}
\hline Végétation & $\begin{array}{c}\mathrm{Fe}_{2} \mathrm{O}_{;} \text {total } \\
\% o \text { mat. min. }\end{array}$ & $\begin{array}{c}\mathrm{Fe}_{2} \mathrm{O}_{3} \text { libre } \\
\% o \text { mat. min. }\end{array}$ & $\begin{array}{l}\text { Taux d'altération } \\
\frac{\mathrm{Fe}_{2} \mathrm{O}_{3} \text { libre }}{\mathrm{Fe}_{2} \mathrm{O}_{3} \text { total }} \times 100\end{array}$ \\
\hline Fétuque & 58,2 & 20.7 & 36 \\
\hline Canche & 69,9 & 14,2 & 20 \\
\hline Myrtille $\ldots$. & 54,3 & 11,3 & 21 \\
\hline
\end{tabular}

(1) Les taux d'altération sont calculés en prenant le fer comme élément de référence, c'est-à-dire l'élément le moins mobile dans les sols étudiés (cf. fig. 1). 
finalement sur les sols dont les horizons supérieurs sont les micux pourvus en argile et en fer libre. Une analyse plus approfondie des caractéristiques organo-minérales de ce; sols ocreilx a fait l'objet d'une autre publication (Boudot \& Bruckert, 1978). Il convient d'en retenir que la plus grande altération observéc sous canche n'est en aucun cas assimilable à une micropodzolisation de surface, mais représente simplement une accentuation de l'argilisation de la roche. Il n'y a aucune destruction d'argile ni migration de complexes organo-minéraux, car l'abondance des éléments phylliteux et métalliques issus de l'argilisation s'y oppose.

Cette évolution conduit à une situation inverse de celle observée sur granite, matériau beaucoup plus sensible à la podzolisation.

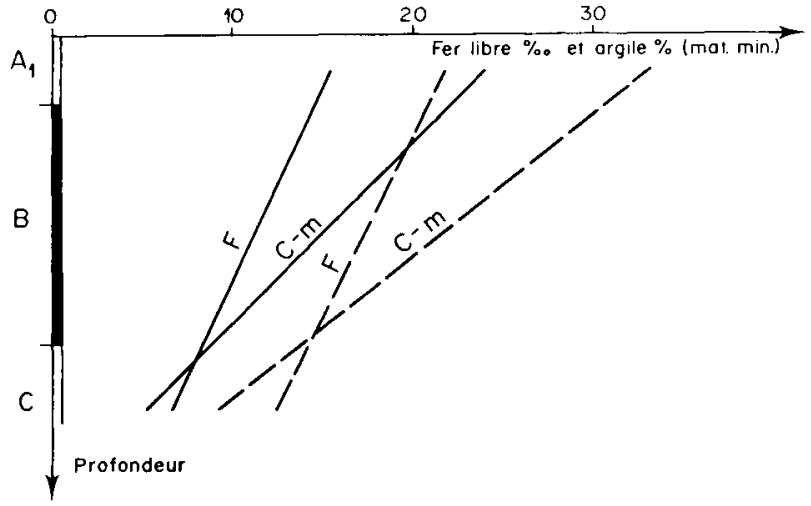

FIG, 1

Distribution de largile (trait plein) et du fer libre (tiretet) dans les sols sous fétuque $(F)$ et sous canche-myrtille $(c-m)$

Répartition of clays (-) and of free iron (- - ) in the soils under Festuca silvatica $(F)$

and under Deschampsia flexuosa-Vaccinium myrtillus $(c-m)$

A l'étage de la hêtraie culminale, les sols bruns acides humifères sont la règle dans les stations en topographie sub-horizontale. Ici, la résistance d'un matériau ancien, peu rajeuni par solifluxion ou érosion au Wurm (effet de protection de la calotte glaciaire, immobile) est fréquemment un facteur essenticl, comme l'a constaté Brethes (1973) dans les vieilles altérites du Morvan.

Par ailleurs, l'affleurement d'une strate géologique schisteuse et la formation d'altération qui en dérive, plus riche en argile et limons fins, offrent les mêmes conditions de brunification.

Sur les pentes raides, par contre, le matériau est rajeuni et, sous mégaphorbiée, on observe des sols bruns ocreux à mull-moder, comme sous la hêtraic sapinière située plus bas (mais masqués par un caractère plus humifère). Sous myrtille, on rencontre des sols ocre podzoliques humifères à moder-mor. La redistribution des complexes organo-ferriques y est excessivement réduite et n'excède pas quelques centimètres. Leur extension est assez faible. 


\section{Tableau 4}

Composition floristique de la hêtraie culminale

Floristic composition of the culminant Beech-grove

\begin{tabular}{|c|c|c|c|c|}
\hline \multirow[b]{2}{*}{ Espèces } & \multicolumn{4}{|c|}{ Indices d'abondance-dominance extrêmes } \\
\hline & $\begin{array}{c}\text { Acero-fagetum } \\
\text { myrtilletosum }\end{array}$ & $\begin{array}{c}\text { A.F. } \\
\text { stellarietosum }\end{array}$ & $\begin{array}{c}\text { A.F. } \\
\text { cicerbitosum }\end{array}$ & $\begin{array}{c}\text { A.F. poaetosum } \\
\text { chaixii }\end{array}$ \\
\hline \multicolumn{5}{|l|}{ Strate arborescente } \\
\hline Fagus silvatica $\ldots \ldots \ldots \ldots$ & 5 & 4 à 5 & $4 \grave{a} 5$ & 5 \\
\hline Acer pseudo-platanus .... & 0 à 2 & 0 à 3 & $+\grave{a} 2$ & 0 à 2 \\
\hline Sorbus aucuparia $\ldots \ldots \ldots$ & 0 à 2 & 0 à 1 & & 0 à 1 \\
\hline \multicolumn{5}{|l|}{ Strate herbacée } \\
\hline Vaccinium myrtillus .... & $2 \times 5$ & & & \\
\hline Maianthemum fibolium & 0 à 2 & & & \\
\hline Stellaria nemorum ..... & 0 à 2 & $+\mathrm{a} 3$ & 0 à 5 & \\
\hline Athyrium filix femina... . & 0 à 2 & 0 à 2 & 2 à 5 & $0 \grave{\mathbf{a}}+$ \\
\hline Adenostyles albifrons ....... & 0 à 2 & $0 \grave{x} 1$ & 2 & $0 \grave{a} 1$ \\
\hline Mulgedium alpinum $\ldots \ldots \ldots$ & 0 à 1 & & 0 à 5 & \\
\hline Athyrium alpestre ... & 0 à 1 & & 0 à 5 & \\
\hline Impatiens nolitangere & & & 0 à 5 & \\
\hline Poa chaixi $\ldots \ldots \ldots$ & 0 à 1 & & 0 à 5 & 3 à 5 \\
\hline Oxalis acetosella $\ldots .$. & 0 à 4 & 0 à 4 & 0 à 2 & 0 à 4 \\
\hline Deschampsia flexuosa & $0 \grave{a}+$ & & & 0 à 2 \\
\hline Polygonatum verticilat. & 1 à 2 & 1 à 2 & 0 à 2 & 0 à 2 \\
\hline Milium effusum $\ldots \ldots \ldots$ & 0 à 2 & $2 \grave{a} 5$ & 0 à 2 & 2 à 3 \\
\hline Phegopteris dryopteris. & 0 à 3 & 0 à 1 & 0 à 2 & \\
\hline Dryopteris dilatata .... & 0 à 2 & 0 à 2 & 0 à 2 & \\
\hline Rumex arifolius $\ldots . \ldots \ldots$. & 0 à 3 & 0 à 3 & $0 \grave{a} 2$ & 0 à 2 \\
\hline Prenanthes purpurea ....... & 0 à 2 & 0 à 3 & 0 à 5 & \\
\hline Luzula albida...$\ldots$. & 0 à 1 & 0 à 1 & 0 à 2 & 1 à 3 \\
\hline Lysimachia nemorum & 0 à 2 & $0 \grave{a} 1$ & 0 à 1 & \\
\hline Rubus idaeus ...... & 0 à 2 & $0 \grave{a} 3$ & $0 \grave{a}+$ & $0 \grave{a}+$ \\
\hline Soligado virga aurea $\ldots \ldots \ldots$ & 0 à 2 & & 0 à 2 & 0 à 2 \\
\hline Lamium galeobdolon ....... & 0 à 2 & $+\grave{a} 2$ & 0 à 3 & + à 1 \\
\hline Luzula maxima $\ldots \ldots \ldots \ldots$ & 0 à 1 & & & 0 à 2 \\
\hline Dryopteris filix mas ... & 0 à 1 & 0 à 1 & 0 à 2 & \\
\hline Lonicera nigra $\ldots \ldots \ldots \ldots$ & 0 à 2 & $0 \grave{a}+$ & & \\
\hline Hieracium silvaticum $\ldots \ldots \ldots$ & $0 \grave{a}+$ & 0 à 1 & & 0 à 2 \\
\hline Paris quadrifolia $\ldots \ldots \ldots \ldots$ & 0 à 1 & 0 à 1 & 0 à 1 & \\
\hline Asperula odorata...$\ldots \ldots$ & & 0 à 4 & 0 à 2 & 0 à 3 \\
\hline Lycopodium annotinum ..... & 0 à 1 & & & \\
\hline
\end{tabular}


Les rankers cryptopodzoliques humifères occupent les sommets des HautesVosges : ce sont des climax climatiques qui, développés aussi bien sur grauwacke que sur granite, caractérisent en fait les hautes chaumes naturelles des massifs océaniques (Duchaufour, 1961). Leur répartition par comparaison avec les sols forestiers qui les entourent, a permis de délimiter l'extension des hautes chaumes primaires (CARBIENER, 1963, 1964, 1966). L'association végétale relevée sur grauwacke est comme sur granite une lande à éricacées (Pulsatillo-micrantae nardetum Tx 37 Vaccinietosum Carb. 66) (tableau 5).

\section{TABleau 5}

Composition floristique des hautes chaumes primaires (sommet du Grand Ballon) Floristic composition of the primary culminant heath (at the top of the «Grand Ballon»)

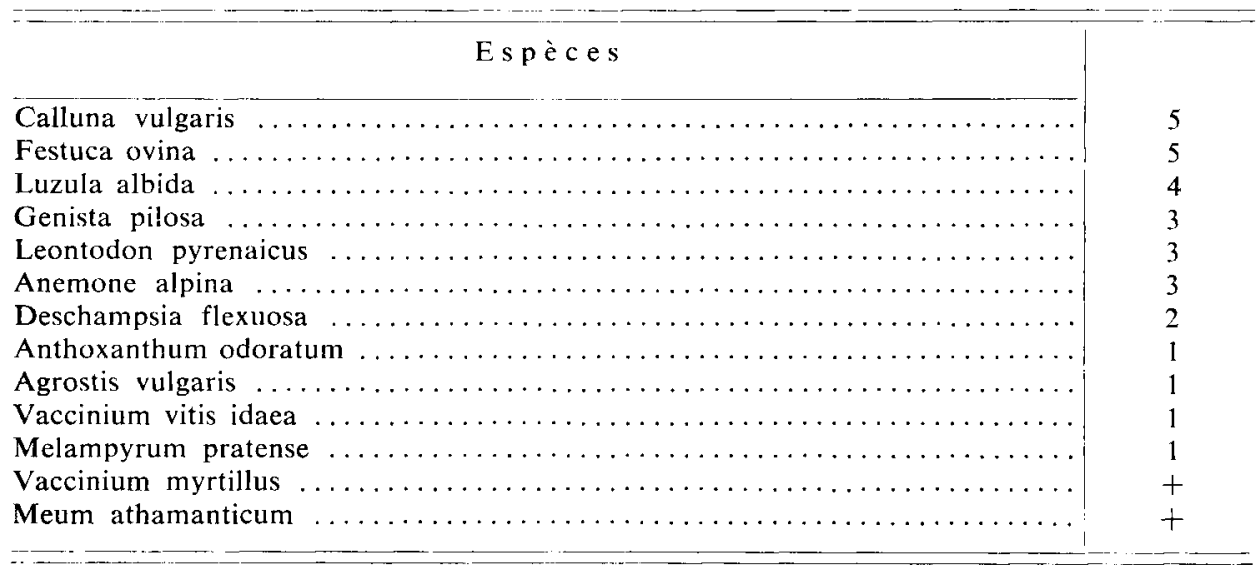

\section{B. - Les climax stationnels}

Les sols bruns colluviaux et les sols colluviaux se développent assez largement dans ces paysages où les ravins d'origine glaciaire sont fréquents. L'effet de colluvionnement sur le pédoclimat, l'activité biologique, l'humification et le cycle biogéochimique des éléments minéraux (Duchaufour et al., 1971 ; Vedy, 1973 ; Guil.lemin et al., 1976) se traduit par le développement de stations à mull très actif caractérisées par l'association de l'érable sycomore (Acer pseudo-platanus), de l'orme des montagnes (Ulmus Montana) et du frêne (Fraxinus exelsior). Bien qu' l'effet du climat soit secondaire, la variation de la composition floristique avec latitude est loin d'être négligeable (tableau 6). Sur ces colluviums, le tilleul (Tilia platyphyllos) et l'alisier blanc (Sorbus aria) ne se trouvent qu’à l'étage du chêne (Fraxino-aceretum sorbetosum ariae, OBERD et al., 1967). A l'étage du sapin apparaissent la monnaie du pape (Lunaria Rediviva) et la ficaire (Ficaria Ranunculö̈des) [Ulmo-aceretum Issl. (24) 26 , s.a. ficarietosum s.a. Nov.]. A l'étage de la hêtraie culminale se rencontre la campanule à larges feuilles (Campanula Latifolia) dans ses rares stations vosgiennes, tandis que la ficaire disparaît [Ulmo-aceretum Issl. (24) 26, s.a. campanuletosum latifoliae s.a. Nov.) (Boudot, 1976). 


\section{Tableau 6}

\section{Composition floristique des stations d'éboulis et de ravin}

I. Fraxino Aceretum Phyllitidetosum (éboulis étage hêtraie sapinière)

II. Fraxino Aceretum Sorbetosum Ariae (ravins étage chênaie)

III. Ulmo Accrelum Ficarietosum (ravins étage hêtraie-sapinière)

IV. Ulmo Aceretum Campanuletosum Latifoliae (ravins étage hêtraie-culminale)

Floristic composition of the stations on screes and in ravines

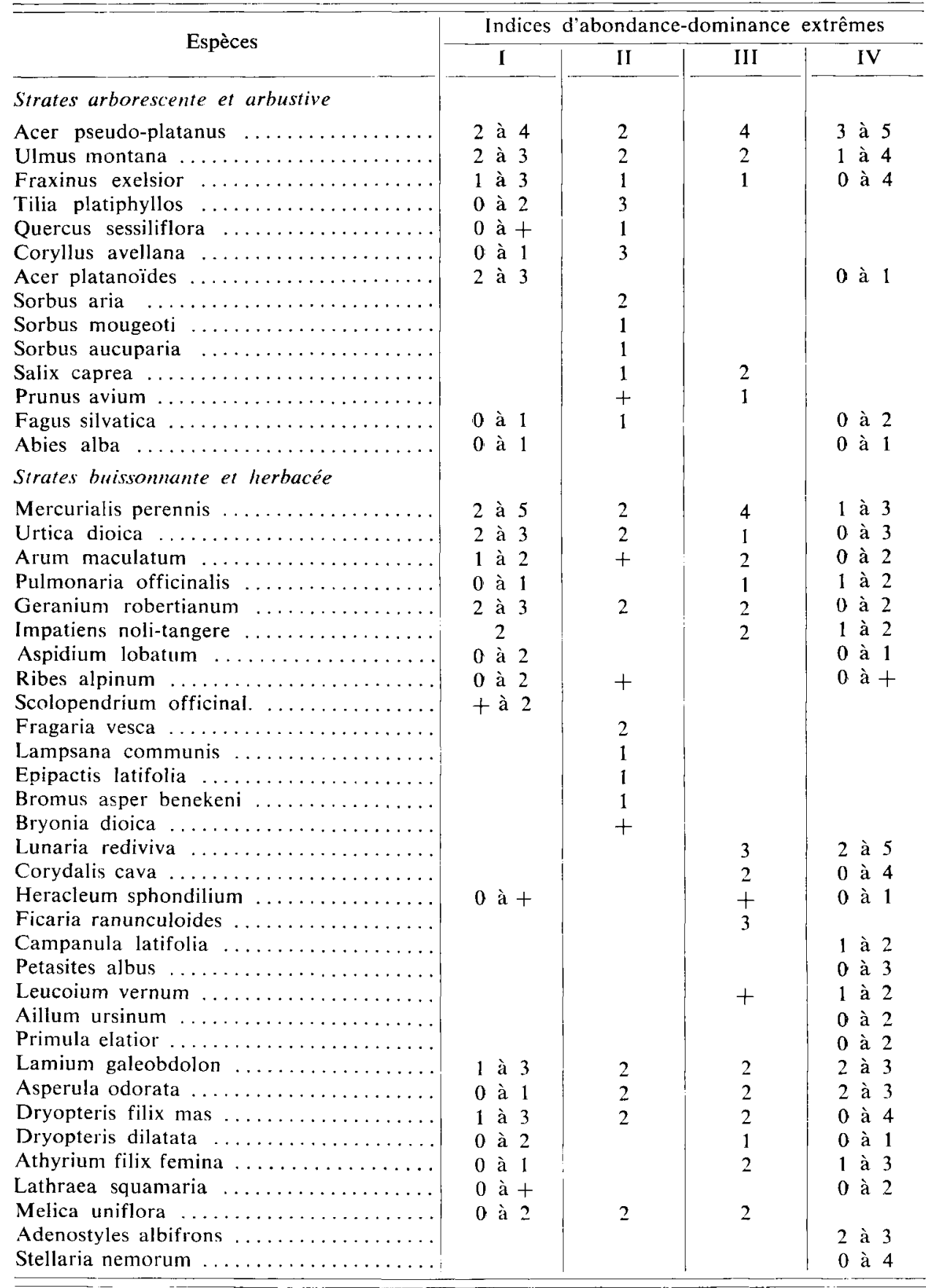


La végétation de ces climax stationnels est donc constituée de groupements d'espèces à exigences trophiques élevées, qui concurrencent efficacement, sur ces terrains argileux propices à l'enrichissement en bases et à une alimentation en eau et en azote optimale, les espèces des climax climatiques avoisinants.

Les sols humiques eutrophes sur éboulis sont des climax stationnels nouveaux caractérisés, à l'étage de la hêtraie-sapinière, par une association identique à celle que l'on rencontre sur les sols humo-calciques ou humo-calcaires d'éboulis carbonatés (Bottner, 1976; Duchaufour et al., 1976) : l'érablière à tilleul et à scolopendre [Fraxino-aceretum phyllitidetosum (Moor 52), ОвеRD et al., 1967] (tableau 5). Leur profil à forte pierrosité comprend un horizon $A_{1}$ très organique et à large structure, qui s'infiltre profondément à l'état discontinu entre les blocs.

La convergence des humus avec ceux des éboulis calcaires est très nette. Il s'agit donc d'un type de climax correspondant à un écosystème dont l'installation et le fonctionnement est particulièrement remarquable sur roche acide. C'est un magnifique exemple de convergence des humus 't de sols analogues au sens de Pallman, que lon observe à la fois sur éboulis de roche-mère acide et sur pierriers calcaires.

\section{IV. - Discussion et conclusion}

Sur grauwacke comme sur granite, le climat des Hautes-Vosges détermine les types dominants de végétation, hêtraie-sapinière, hêtraie culminale et hautes chaumes. A chacun de ces étages climatiques, correspond un sol climax (climax climatique), soit tout à fait analogue au sol du même étage sur granite (ranker cryptopodzolique à l'étage des hautes chaumes), soit présentant quelque différence avec le sol correspondant sur granite, comme lindique le tableau 7.

A l'étage de la hêtraie-sapinière, les profils pédologiques ont une couleur ocre plus ou moins intense et une structure «foisonnante» en microagrégats, caractéristique des sols bruns ocreux.

A l'étage de la hêtraie culminale, la composante ocre des profils śatténue ou disparaît. Le sol est le plus souvent brun acide plus ou moins humifère ou ocre podzolique.

A ces deux étages, la redistribution du fer et de l'aluminium est exceptionnelle: il est clair que le matériau grauwacke, extrêmement altérable. excrec un fort pouvoir tampon vis-à-vis de la podzolisation. On sait en effet qu'il faut une quantité de matière organique excédentaire par rapport à la capacité d’absorption du support minéral des profils pour qu'une redistribution d'éléments métalliques puisse se réaliser sous l'effet d'une géochimic organique acide (Zzbrowski, 1973; Heilman \& Goss, 1974 ; Hetier, 1976). Ce n'est qu'à l'étage des hautes chaumes que la matière organique provoque une redistribution de l'aluminium dans les rankers cryptopodzoliques (podzolisation climatique).

Ainsi, le matériau grauwacke, par sa composition chimique et minérale, freine la différenciation pédogénétique. Il en résulte une certaine uniformisation des types de sols climatiques situés à une altitude donnée. 
Tableau 7

Comparaison des types de sols par station sur grauwacke et sur granite Comparison between the different soils for each station on graywacke and on granite

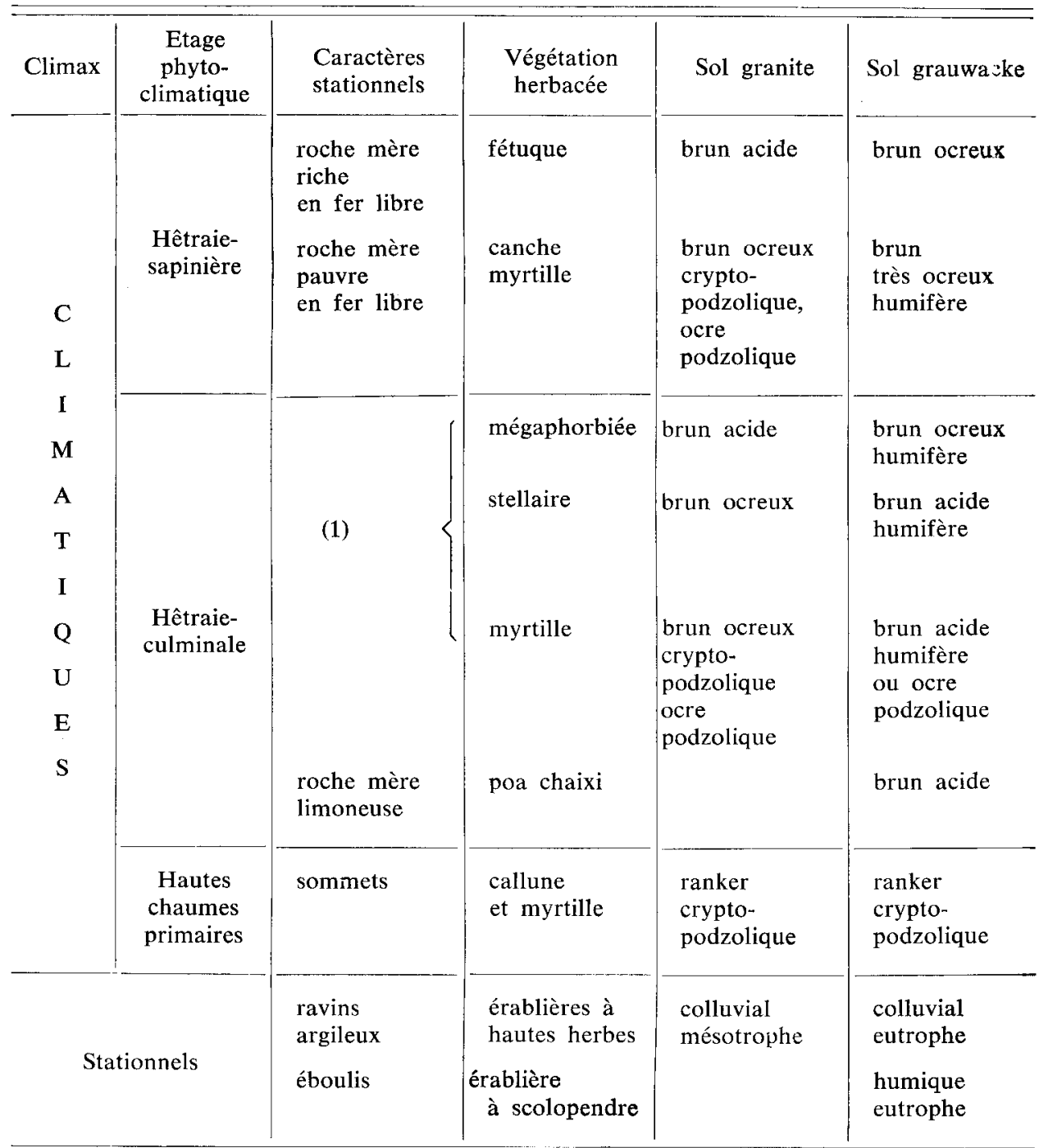

(1) Sur granite le facteur de différenciation est la richesse en fer de l'arène, sur grauwacke c'est la situation topographique ou géomorphologique, et le microclimat : voir tableau 1.

Par contre, à l'intérieur de chaque étage de végétation, on constate une diversification des climax stationnels remarquables, et pour certains, très originaux :

- l'Ulmo aceretum ficarietosum occupe les sols colluviaux eutrophes de ravin à l'étage de la hêtraie-sapinière. Il est remplacé par l'Ulmo aceretum campanulesotum latifoliae à l'étage de la hêtraie culminale; 
- le Fraxino aceretum phyllitidetosum colonise les pierriers mobiles et les blocailles humides dépourvues de fraction minérale fine de l'étage de la hêtraiesapinière; le profil pédologique est un sol humique eutrophe d'éboulis. Ce groupement est également remplacé par l'Ulmo-aceretum campanuletosum latifoliae à l'étage de la hêtraie culminale.

Ces climax stationnels formés sur roche acide portent tout un cortège d'herbacées fortement indicatrices de la richesse en bases du sol, notamment en calcium échangeable concentré dans les humus par le jeu du cycle biogéochimique (VEDY, 1973; Guillemin, 1976) et en azote minéral $\left(\mathrm{NH}_{4}\right.$ et $\left.\mathrm{NO}_{3}\right):$ Mercurialis perennis, Urtica dioica (qui a ici ses stations primaires), Lunaria rediviva, Corydalis cava, parfois la très discrète Lathraea squamaria..., en sont les plus importantes.

Reçu pour publication en octobre 1980. 


\section{Annexe}

A. - Caractérisation des stations

\section{I. - Climax climatiques}

a) Sol brun ocreux sous hêtraie-sapinière à fétuque

\begin{tabular}{|c|c|c|c|c|}
\hline $\begin{array}{c}\text { Horizon } \\
\text { Profondeur en } \mathrm{cm}\end{array}$ & $\mathrm{pH}$ & $\begin{array}{l}\mathrm{T} \text { me } \\
100 \mathrm{~g}\end{array}$ & $\frac{\mathrm{S}}{\mathrm{T}} \times 100$ & $\mathrm{C} / \mathrm{N}$ \\
\hline$A_{1}(0-2(10))($ mull-moder $) \ldots \ldots \ldots$ & 4,2 & 41,1 & 22,1 & 20.4 \\
\hline$A_{3}(2(10)-55)$ & 4,35 & 18.1 & 4,4 & 23,5 \\
\hline (B) $(55-90) \ldots$ & 4,6 & 12,4 & 7,3 & 23,3 \\
\hline (B) $/ C$ (inf. 90$)$ & 5,3 & 10,3 & 11,6 & - \\
\hline
\end{tabular}

\begin{tabular}{|c|c|c|c|c|c|c|c|c|c|}
\hline \multirow{2}{*}{ Horizon } & \multirow{2}{*}{$\begin{array}{r}\text { M.O. } \\
(\%)\end{array}$} & \multicolumn{5}{|c|}{ Grunolométrie $(\%)$} & \multicolumn{3}{|c|}{ Sesquioxydes libres $(\%)$} \\
\hline & & A & LF & LG & SF & SG & $\mathrm{Fe}$ & $\mathrm{Al}$ & $\mathrm{Si}$ \\
\hline $\mathrm{A}_{1}$ & 30,3 & 18,5 & 14,9 & 7,7 & 10,1 & 18,5 & 13,7 & 6,5 & 2,0 \\
\hline $\mathrm{A}_{3}$ & 7,3 & 11,1 & 24,2 & 11,9 & 16,5 & 29,0 & 15,2 & 12,7 & 3,0 \\
\hline (B) & 2,9 & 3,9 & 22,4 & 12,4 & 18,7 & 34.2 & 15,0 & 14,7 & 4.7 \\
\hline (B) $/ \mathrm{C} \ldots$ & - & - & - & - & - & - & 14,2 & 12,5 & 4,2 \\
\hline
\end{tabular}

Remarque : Structure d'ensemble à tendance floconneuse; couleurs brun-ocracée en (B). 
b) Sol brun ocreux sous hêtraie-sapinière à canche flexueuse

\begin{tabular}{|c|c|c|c|c|}
\hline $\begin{array}{c}\text { Horizon } \\
\text { Profondeur en } \mathrm{cm}\end{array}$ & $\mathrm{pH}$ & $\begin{array}{l}\mathrm{T} \text { me } \\
100 \mathrm{~g}\end{array}$ & $\frac{S}{T} \times 100$ & $\mathrm{C} / \mathrm{N}$ \\
\hline$A_{1}(0-3(5))($ moder $) \ldots \ldots \ldots \ldots \ldots$ & 4,1 & 29,0 & 18,1 & 19,0 \\
\hline$A_{1}-B_{11}(3(5)-22) \ldots \ldots \ldots \ldots \ldots \ldots$ & 4,35 & 21,0 & 3,8 & 20,7 \\
\hline $\mathrm{B}_{\mathrm{h}}-\mathrm{B}_{\mathrm{s}}(22-38)$ & 4,7 & 19,0 & 3,1 & 23,3 \\
\hline $\mathbf{B}_{\mathrm{s}}(38-60) \ldots$ & 4,75 & 13,2 & 3,9 & 23,6 \\
\hline$B / C(60-80) \ldots$. & 5,1 & 8,8 & 5,7 & 23,3 \\
\hline C & 5,3 & 4,8 & 10,0 & 14,5 \\
\hline
\end{tabular}

Remarque : Structure d'ensemble très floconneuse et couleur très rouille vif en $B_{s}$; argile piégé en faux-limons.

\begin{tabular}{|c|c|c|c|c|c|c|c|c|c|}
\hline \multirow{2}{*}{ Horizon } & \multirow{2}{*}{$\begin{array}{l}\text { M.O. } \\
(\%)\end{array}$} & \multicolumn{5}{|c|}{ Granulométrie (\%) } & \multicolumn{3}{|c|}{ Sesquioxydes libres $(\%)$} \\
\hline & & A & $\mathrm{LF}$ & LG & SF & SG & $\mathrm{Fe}$ & $\mathrm{Al}$ & $\mathrm{Si}$ \\
\hline$A_{1} \ldots$ & 27,2 & 15,8 & 17,4 & 7,8 & 11,0 & 20,8 & 19,8 & 7,0 & 2,1 \\
\hline$A_{1}-B_{11} \cdots$ & 10,8 & 19,3 & 22,7 & 9,5 & 13,9 & 23,8 & 29,9 & 11,3 & 2,0 \\
\hline$B_{11}-B_{s} \ldots$ & 12,2 & 14,8 & 26,1 & 12,3 & 12,5 & 22,6 & 28,5 & 13,7 & 1,7 \\
\hline $\mathrm{B}_{\mathrm{s}} \ldots$ & 6,2 & 11,5 & 22,7 & 11,6 & 13,3 & 34,6 & 21,0 & 14,1 & 2,7 \\
\hline $\mathrm{B} / \mathrm{C}$ & 3,5 & 7,4 & 18,6 & 12,0 & 11,5 & 47,0 & 18,0 & 13,5 & 2,5 \\
\hline $\mathrm{C} \ldots \ldots$ & 0,8 & 4,2 & 8,7 & 3,3 & 15,6 & 67,5 & 9,8 & 6,3 & 3,2 \\
\hline
\end{tabular}


c) Sol brun acide sous hêtraie culminale à millet et stellaire

\begin{tabular}{|c|c|c|c|c|}
\hline $\begin{array}{c}\text { Horizon } \\
\text { Profondeur en } \mathrm{cm}\end{array}$ & $\mathrm{pH}$ & $\begin{array}{l}T \text { me } \\
100 \mathrm{~g}\end{array}$ & $\frac{\mathrm{S}}{\mathrm{T}} \times 100$ & $\mathrm{C} / \mathrm{N}$ \\
\hline$A_{1}(0-7)$ (mull-moder) .. & 4,1 & 26,6 & 14,5 & 15,5 \\
\hline$A_{: 3}(7-40)$ & 4,4 & 15,2 & 4,3 & 14,8 \\
\hline (B) $(40-65)$ & 4,7 & 13,0 & 4,0 & 15,5 \\
\hline$C$ inf. $65 \ldots$ & 5,05 & 8,4 & 4,9 & 13,2 \\
\hline
\end{tabular}

\begin{tabular}{|c|c|c|c|c|c|c|c|c|c|}
\hline \multirow{2}{*}{ Horizon } & \multirow{2}{*}{$\begin{array}{l}\text { M.O. } \\
(\%)\end{array}$} & \multicolumn{5}{|c|}{ Granulométrie $(\%)$} & \multicolumn{3}{|c|}{ Sesquioxydes libres $(\%)$} \\
\hline & & $\mathbf{A}$ & LF & LG & SF & SG & $\mathrm{Fe}$ & $\mathrm{Al}$ & $\mathrm{Si}$ \\
\hline$A_{1}$ & 20,5 & 13,9 & 19,3 & 9,7 & 15,9 & 20,7 & 17,7 & 6,7 & 3,1 \\
\hline $\mathrm{A}_{3}$ & 7,3 & 14,6 & 23,3 & 9,5 & 17,8 & 27,4 & 18,6 & 8,0 & 2,8 \\
\hline (B) & 4,7 & 13,1 & 28,9 & 12,8 & 18,7 & 21,8 & 18,0 & 9,5 & 2,9 \\
\hline $\mathrm{C}$ & 1,8 & 9,1 & 26,1 & 15,2 & 21,9 & 25,9 & 14,4 & 6,8 & 1,7 \\
\hline
\end{tabular}

Remarque : Structure d'ensemble sub-polyédrique; couleur brune. 
d) Ranker cryptopodzolique des hautes chaumes sous lande à callune

\begin{tabular}{|c|c|c|c|c|}
\hline $\begin{array}{c}\text { Horizon } \\
\text { Profondeur en } \mathrm{cm}\end{array}$ & $\mathrm{pH}$ & $\begin{array}{l}\mathrm{T} \text { me } \\
100 \mathrm{~g}\end{array}$ & $\frac{\mathrm{S}}{\mathrm{T}} \times 100$ & $\mathrm{C} / \mathrm{N}$ \\
\hline$A_{11}(0-25)$ (moder-mor) $\ldots$ & 4,45 & 37,7 & 6,3 & 16,9 \\
\hline $\mathrm{A} / \mathrm{B}_{1}(25-45) \ldots$ & 4,7 & 38,8 & 3,8 & 14,7 \\
\hline $\mathrm{A} / \mathrm{B}_{2}(45-80)$ & 5,2 & 25,1 & 4,9 & 13,7 \\
\hline $\mathrm{B} / \mathrm{C}$ (inf. 80 ) $\ldots$ & 5,1 & 10,8 & 8,4 & 14,4 \\
\hline
\end{tabular}

\begin{tabular}{|c|c|c|c|c|c|c|c|c|c|}
\hline \multirow{2}{*}{ Horizon } & \multirow{2}{*}{$\begin{array}{l}\text { M.O. } \\
\text { (\%) }\end{array}$} & \multicolumn{5}{|c|}{ Granulométrie $(\%)$} & \multicolumn{3}{|c|}{ Sesquioxydes libres $(\%)$} \\
\hline & & A & LF & LG & $\mathrm{SF}$ & SG & $\mathrm{Fe}$ & Al & $\mathrm{Si}$ \\
\hline$A_{0}$ & 23,7 & 20,1 & 18,8 & 10,7 & 9,9 & 16,8 & 24,2 & 17,1 & 2,7 \\
\hline $\mathrm{A} / \mathrm{B}_{1}$ & 20,3 & 19,8 & 21,8 & 10,6 & 10,3 & 17,2 & 19,7 & 26,3 & 2,7 \\
\hline $\mathrm{A} / \mathrm{B}_{2}$ & 12,6 & 14,0 & 23,6 & 19,2 & 15,3 & 15,1 & 18,8 & 27,4 & 5,9 \\
\hline $\mathrm{B} / \mathrm{C}$ & 3,8 & 7,7 & 19,9 & 17,0 & 17,0 & 34,5 & 14,4 & 13,4 & 4,1 \\
\hline
\end{tabular}

Remarque : Structure très floconneuse; couleur brun foncé à noir; argile piégé en fauxlimons. 


\section{II. - Climax stationnels}

a) Sol colluvial à mull eutrophe sous érablière à hautes herbes

\begin{tabular}{|c|c|c|c|c|}
\hline $\begin{array}{c}\text { Horizon } \\
\text { Profondeur en } \mathrm{cm}\end{array}$ & $\mathrm{pH}$ & $\begin{array}{l}\mathrm{T} \mathrm{me} \\
100 \mathrm{~g}\end{array}$ & $\frac{S}{T} \times 100$ & $\mathrm{C} / \mathrm{N}$ \\
\hline$A_{1},(0-5)$ (mull) & 5,65 & 16,9 & 77,3 & 13,1 \\
\hline$A_{1.2}(5-20)$ & 5,7 & 17,1 & 74,0 & 12,3 \\
\hline$C(75-110) \ldots$ & 5,3 & 10,0 & 47,1 & 9,7 \\
\hline
\end{tabular}

\begin{tabular}{|c|c|c|c|c|c|c|c|c|c|}
\hline \multirow{2}{*}{ Horizon } & \multirow{2}{*}{$\begin{array}{l}\text { M.O. } \\
(\%)\end{array}$} & \multicolumn{5}{|c|}{ Granulométrie (\%) } & \multicolumn{3}{|c|}{ Sesquioxydes libres $(\% o)$} \\
\hline & & $A$ & LF & LG & SF & SG & $\mathrm{Fe}$ & $\mathrm{Al}$ & $\mathrm{Si}$ \\
\hline $\mathbf{A}_{1.1}$ & 9,2 & 19,1 & 18,1 & 10,2 & 16,4 & 27,1 & 10,8 & 5,5 & 1,5 \\
\hline$A_{1.2}$ & 7,5 & 21,5 & 18,0 & 9,2 & 16,7 & 27,0 & 11,4 & 5,1 & 1,7 \\
\hline $\mathrm{C}$ & 2,3 & 22,0 & 20,8 & 10,6 & 15,0 & 29,2 & 13,5 & 6,6 & 2,0 \\
\hline
\end{tabular}

Remarque : Structure polyédrique; couleur brune; argile bien exprimée. 
b) Sol humique eutrophe d'éboulis sous érablière à tilleul et scolopendre

\begin{tabular}{c|c|c|c|c}
\hline $\begin{array}{c}\text { Horizon } \\
\text { Profondeur en cm }\end{array}$ & $\mathrm{pH}$ & $\begin{array}{l}\mathrm{T} \text { me } \\
100 \mathrm{~g}\end{array}$ & $\frac{\mathrm{S}}{\mathrm{T}} \times 100$ & $\mathrm{C} / \mathrm{N}$ \\
\hline $\mathrm{A}_{1.1}(0-10)$ (mull humifère) $\ldots \ldots \ldots$ & 6,9 & 104,8 & 100 & 13,8 \\
$\mathrm{~A}_{1.2}(10-60) \ldots \ldots \ldots \ldots \ldots \ldots \ldots \ldots$ & 7 & 110,4 & 100 & 14,8 \\
\hline
\end{tabular}

\begin{tabular}{|c|c|c|c|c|}
\hline \multirow{2}{*}{ Horizon } & \multirow{2}{*}{$\begin{array}{l}\text { M.O. } \\
(\%)\end{array}$} & \multicolumn{3}{|c|}{ Sesquioxydes libres (\%o) } \\
\hline & & $\mathrm{Fe}$ & $\mathrm{Al}$ & $\mathrm{Si}$ \\
\hline $\mathbf{A}_{1.1}$ & 89 & 6,3 & 4,1 & 0,2 \\
\hline $\mathrm{A}_{1.2}$ & 74 & $11 ; 4$ & 7,1 & 1,0 \\
\hline
\end{tabular}

Remarque : Structure bien construite en petits agrégats; couleur noire; profil humifère par déficit de la phase minérale. 


\author{
Summary \\ Vegetation and climax-soils on Graywackes of the Markstein sery \\ (Vosges, France)
}

On the Graywackes of Markstein sery (Vosges), the influence of the climate on vegetation and on the virtual orientation of pedogenesis is the sam that in granitic Hautes-Vosges. Thus, we can observe on these materials, important pedogenetic divergences with the evolution on granitic parent materials, at the same climatic level. The climatic altitudinal sequence of the climax soils developed on graywackes parent materials is as follows :

《ochrous brown soil » at the «Sapinière-Hêtraie » level (Abies pectinata-Fagus silvatica) (acid brown soil on granitic parent materials),

«acid brown soil» at the culninal «Hêtraie» level (Fagus silvatica) (ochrous brown soil ond ochrous podzolic soil on granitic rocks),

"cryptopodzolic ranker» at the high-prairies level (Hautes Chaumes).

On graywackes parent materials, the pedogenetic evolution is less marked than on granitic parent materials. On these last materials, the term of ochrous podzolic soils sometimes occurs. Beyond the altitudinal repartition of soils is inversed at the forest level where the altitudinal sequence «Acid brown soil-Ochrous soil-cryptopodzolic soil» is observed on granitic rocks. Only the last type of soil is the same for both of materials.

\title{
Références bibliographiques
}

BOTTNER P., 1971. La pédogénèse sur roches mères calcaires dans une séquence bioclimatique méditerranéo-alpine du Sud de la France. Thèse Doctorat d'Etat, Montpellier, 278 pages.

Boudot J.-P., 1976. Ecologie du paysage et processus de pédogénèse sur les Grauwackes de la série du Markstein (Hautes-Vosges.) Thèse Doct. Spéc. Sci. Biol. (Ecologie). Université Louis Pasteur, Strasbourg.

BoudoT J.-P., 1978. Sur les interactions au sein des écosystèmes naturels : relations entre climat, géomorphologie, géologie, pédologie et végétation dans les Vosges. Bull. Soc. Ind. de Mulhouse, 3, 13-33.

Boudot J.-P. et BRuCKERT S., 1978. Complexes organo-métalliques et structures microagrégées des sols sur matériaux chloriteux du système schisto-grauwackeux vosgien. Science du Sol, Bull. A.F.E.S., 1, 31-40.

Brethes A., 1973. Mode d'altération et différenciation pédogénétique sur leucogranites du Massif du Morvan, comparaison avec le Massif Vosgien. Thèse Doct. Spéc., Nancy, 97 p. Acad. Sci., 20 avril 1964, 258, 4136, 4138.

Carbiener R., 1963. Remarques sur un type de sol encore peu étudié : le Ranker Cryptopodzolique de l'étage subalpin des massifs hercyniens français. C.R. Acad. Sciences, Paris, 256, 977-979.

Carbiener R., 1963. Les sols du massif du Hohneck - Leurs rapports avec le tapis végétal. Le Hohneck, Ass. philom. Als. Lorr., Strasbourg, 103-152.

Carbiener R., 1964. La détermination de la limite naturelle de la forêt par des critères pédologiques et géomorphologiques dans les Hautes-Vosges et le Massif Central. C.R. Acad. Sciences, 20 avril 1964, 258, 4136, 4138.

Carbiener R., 1966. La végétation des Hautes-Vosges dans ses rapports avec les climats locaux, les sols et la géomorphologie. Thèse Fac. Sciences, Paris, 112 p., dactylographiée.

Duchaujour Ph., 1961. Les sols humiques silicatés d'altitude. Leur origine, leurs propriétés, leur mise en valeur. R.F.F., 4, 244-250. 
Duchaufour Ph., 1961. Le rôle de la végétation dans l'évolution des sols. Bull. de l'A.F.E.S., 168-193.

Duchaufour Ph., 1970. Humification et Ecologie. Cahier de l'O.R.S.T.O.M., Série Pédologie, VII (4), 379-390.

Duchaufour Ph., Faivre P., Gury M., 1976. Atlas pédologique des sols du monde.

GAGNY C., 1962. Caractères sédimentologiques et pétrographiques des schistes et grauwackes du Culm dans les Vosges méridionales. Bull. Serv. Carte Géol. Als. Lorr., 15 (4), 139-160, Strasbourg.

Guillemin M., Rouiller J., BRuCKert S., 1976. Relations entre groupes écologiques et propriétés physico-chimiques des sols brunifiés des Vosges cristallines. Ann. Sci. Fores., sous presse.

Heilman P.E., Gass L., 1974. Parent materials and chemical properties of mineral soils in South-East Alaska. Soil Sci., 117 (1), 21-27.

Oberdorfer E., Görs, Korneck, Lohmeyer, Muller, Philippi, Seibert, 1967. Systematische Übersicht der Westdeutschen Phanerogamen und Gefasskryptogamen Gesellschaften. Ein Diskussionsentwurf. Schriftenr. $f$. Vegetationskunde, 2, 7-62.

Rivalenti G. et Sighinolfi G.P., 1969. Geochemical study of graywackes as possible material of para-amphibolites. Contrib. mineral. Petrol, 23, pp. 173-188, Heidelberg.

Souchier B., 1971. Evolution des sols sur roches cristallines à l'étage montagnard (Vosges). Mém. Serv. Carte Géol. Als. Lorr., 33, Strasbourg.

VEDY J.-C., 1973. Relations entre le cycle biogéochimique des cations et l'humification en milieu acide. Thèse doct. Etat Univ. Nancy.

Zebrowski Cl., 1973. Quelques andosols de Madagascar et de la Réunion. Problème de la podzolisation sur un andosol. Bull. de liaison du thème $\mathrm{D}$ « andosols 》. Comité Technique Pédologie O.R.S.T.O.M., 2, 53-77. 